\title{
Lack of Relevant Haemogram Changes During Percutaneous Radiotherapy of Localised Prostate Cancer
}

\author{
FELIX ZWICKER $^{1,2}$, BENEDICT SWARTMAN ${ }^{1}$, PETER E. HUBER ${ }^{1,2}$, \\ KLAUS HERFARTH $^{1}$, JÜRGEN DEBUS ${ }^{1,2}$ and HENRIK HAUSWALD ${ }^{1}$ \\ ${ }^{1}$ Department of Radiation Oncology, Heidelberg University Hospital, Heidelberg, Germany; \\ ${ }^{2}$ Clinical Cooperation Unit Molecular Radiation Oncology, \\ German Cancer Research Centre (DKFZ), Heidelberg, Germany
}

\begin{abstract}
Background/Aim: In percutaneous radiotherapy dose-distribution and volumetric-load of normal tissue varies in different radiation-techniques. Haematotoxicity may lead to deficiencies of the immune and blood system or to secondary malignancies. Therefore, regular blood-counts are carried out during fractionated radiotherapy. The aim was to investigate patient haemogram courses during radiotherapy of localised prostate-cancer treated with different radiationtechniques ( $n=3)$. Patients and Methods: In this prospective study, blood count changes were examined during fractionated radiotherapy (3D-conformal-RT/step-and-shootIMRT/helical-IMRT) on the prostate-region in localised prostate-cancer cases $(n=50)$. Results: The whole patient group displayed a small but significant reduction in leukocytes. This reduction was higher in the two IMRT groups compared to the 3D-group but without any case of leukopenia. Haemoglobin- or thrombocyte-levels did not significantly change. Conclusion: Regardless of the delivery mode used, localised fractionated irradiation of prostate region did not cause any clinically relevant haemogram changes in this study. These findings question the necessity of regular blood counts during fractionated radiotherapy of the prostate region for patients without any risk factors.
\end{abstract}

The use of radiation therapy for cancer treatment has two primary goals: a) to effectively combat the tumour tissue and b) to protect the patient's unaffected, normal tissue as much

This article is freely accessible online.

Correspondence to: Felix Zwicker, MD, German Cancer Research Centre (DKFZ) E055, Im Neuenheimer Feld 280, 69120 Heidelberg, Germany. Tel: +49 (0) 6221422616, email: f.zwicker@dkfz.de

Key Words: Prostate cancer, radiotherapy, haemogram, leukocytes, thrombocytes, haemoglobin, helical tomotherapy, IMRT, medical costs. as possible (1-3). The normal tissue or organs that do not require treatment may be close to the target volume of the affected, treated tissue. In general, the closer the two tissue types are, the higher the risk of radiation exposure, and the higher the risk of acute or late organ damage. The quality of radiation and delivery mode varies in curative percutaneous radiation therapy. Gamma rays, electrons, hadrons, and photons (in the form of high-energy X-rays generated by a linear accelerator) are used. A range of delivery modalities are used for percutaneous photon radiation. The traditional mode is three-dimensional (3D) conformal radiation therapy, while the intensity-modulated radiation therapy (IMRT) offers options, such as the step-and-shoot IMRT and helical IMRT (also known as tomotherapy) schemes. These different percutaneous modes are responsible for the different transversal and longitudinal distributions of the integral dose in normal body tissues, which are not the targets of the primary radiation. The integrated transversal dose comprises primary radiation segments, secondary, and tertiary radiation, and small amounts of leakage radiation from the primary/multileaf collimator and the linear accelerator itself. The longitudinal integral dose consists primarily of secondary, tertiary, and leakage radiations from the appliance (4-7). In comparison to the 3D conformal mode, the intensity-modulated radiation therapy mode causes lower medium-dose and high-dose exposure, and increased lowdose exposure in the surrounding normal tissue $(8,9)$.

In general, the higher the integral dose is in relation to the total body volume, the higher the dose exposure is in the haematopoietic bone marrow that is particularly sensitive to radiation. The key factors here are the volume of the tumour to be irradiated and the delivery mode used for the radiation. In particular, fractionated radiation over a long period can potentially cause acute changes to the blood count, associated weakness of the immune system, and increased risk of infection. One possible late side-effect is the increased risk of leukaemia (10). Even a low-dose exposure in normal tissue causes a risk of a secondary malignant tumour (11). 
The concentration of haematopoietic bone marrow in the pelvic region means that it is particularly important to consider these associations when dealing with radiation therapy in this area. For example, in the pelvic region, dosevolume effects increase the haematotoxicity and the risk of viral and bacterial infections $(12,13)$.

The information outlined above explains why it is a standard clinical procedure to carry out blood counts every 1-2 weeks throughout any course of fractionated radiation therapy which lasts several weeks. In this context, Cozzarini et al. carried out a retrospective analysis which showed statistically significant rates of leukocytopenia and thrombocytopenia in patients who underwent fractionated radiation of the prostate region $(70.4 \mathrm{~Gy})$ and of the whole pelvic lymphatic drainage system (50.2 Gy). No statistically significant differences were observed between the different IMRT delivery modes used (static field IMRT, modulated arc therapy, helical IMRT) (14).

Pinkawa et al. compared the irradiation of the whole pelvis in prostate cancer to the irradiation of the prostate region only. In their research, the irradiation of the pelvis led to a statistically significant increase in the rates of leukopenia and anaemia (grade 2) (15). A study by Sanguineti et al. compared leukocyte counts by following hypofractionated versus conventional fractionated radiation treatments for localised prostate cancer. Treatments were delivered with the use of the $3 \mathrm{D}$ conformal mode. The conventional fractionated group displayed a statistically significant leukocyte count drop (16).

For this prospective study, we examined changes to the blood count (haemoglobin, leukocytes, and thrombocytes) in patients who underwent a course of conventional fractionated radiation therapy on the prostate region for localised prostate cancer. Treatment was delivered by one of the following modes: 3D, IMRT, or TOMO. Radiation therapy of the prostate is a frequent indication. Given this indication, the impact on the blood count of different radiation delivery modes that imposes different distributions of the integral dose are of clinical interest.

\section{Patients and Methods}

Patients. Patients were all males and investigated within a singlecentre prospective study. The median age was 70 years (range $=52$ 82 years). In all the studied cases, irradiation of the prostate region was indicated because of a localised prostate carcinoma. This was either a primary radiation therapy of the prostate gland or additive/adjuvant radiation therapy after primary prostatectomy. The exclusion criteria were: the indication for additional radiation of lymphatic regions of the pelvis and prior radiation indicated in the patient's medical history. Fifty patients were recruited in total: 15 for 3D, 15 for IMRT, and 20 for TOMO. All participating patients provided written informed consent. The study was conducted according to the World Medical Association Declaration of Helsinki
Table I. Technical data of the used irradiation techniques: 3D, IMRT and TOMO.

\begin{tabular}{lccc}
\hline & $3 \mathrm{D}$ & IMRT & TOMO \\
\hline Single doses & $2 \mathrm{~Gy}$ & $2 \mathrm{~Gy} / 2.17 \mathrm{~Gy}$ & $2 \mathrm{~Gy} / 2.17 \mathrm{~Gy}$ \\
Cumulative doses & $66-70 \mathrm{~Gy}$ & $70 \mathrm{~Gy} / 76 \mathrm{~Gy}$ & $70 \mathrm{~Gy} / 76 \mathrm{~Gy}$ \\
Boost & Sequential & Integrated & Integrated \\
Beams & 4 & 7 or 9 & Helical \\
Energy & $6 \mathrm{MV}$ photons & $6 \mathrm{MV}$ photons & $6 \mathrm{MV}$ photons \\
IGRT & kilo-voltage & kilo-voltage & mega-voltage
\end{tabular}

and the ICMJE Recommendations for the Protection of Research Participants and was approved by the ethics committee at the Heidelberg University in Germany.

Radiation therapy. Patient treatment was not influenced by the study, and they were arbitrarily assigned to the delivery-mode groups as part of the hospital routine, thus emulating a pseudo randomisation assignment scheme. The clinical target volume (CTV) and the planning target volume (PTV) were determined in accordance with the Institute's guidelines. Planning CT scans were performed with a slice thickness of $3 \mathrm{~mm}$ with a full bladder and an empty rectum. PTV comprised of the prostate gland and seminal vesicle or the prostatic fossa, the bottom part of the bladder, and the anterior rectal wall with a dorsal margin of $0.5 \mathrm{~cm}$ and a margin of $0.8 \mathrm{~cm}$ in other directions. The dose on the anterior rectal wall (1/3 of the total circumference) did not exceed a median value of $70 \mathrm{~Gy}$. Inverse treatment planning for the IMRT group was performed using the KonRad software developed at the German Cancer Research Centre (DKFZ). The planning process for the IMRT treatment has already been described in detail by Schlegel et al. (17). Inverse planning for regular TOMO delivery was performed with the use of the planning software TomoTherapy ${ }^{\circledR}$. For the 3D group, treatment was planned with the use of the software Pinnacle. The planning data provided information on the absolute PTV in each patient. Radiotherapy was performed using the department's linear accelerators: a) Oncor (Siemens, Germany) for 3D and IMRT and b) TomoTherapy ${ }^{\circledR} \mathrm{Hi}$-Art (Accuray, USA) for helical IMRT (TOMO). Radiotherapy courses were performed in 3335 fractions over 7 weeks. The technical parameters and the prescribed doses, which were used for the different radiation delivery modes, are listed in Table I. Transversal dose distributions, which depended on the used radiation technique, are shown in Figure 1. At the end of radiotherapy, no side-effects were observed that exceeded grade 3 (Radiation Therapy Oncology Group). As required by the national guidelines, clinical follow-up after radiation therapy was carried out by each patient's attending urologist.

Blood samples. Blood $(7.5 \mathrm{ml})$ was obtained from peripheral veins from all patients every week during radiotherapy. The first sample was obtained immediately before the first fraction of radiotherapy (initial value). All additional samples were collected in the middle of the treatment period in weeks 2-7. Only patients who attended all blood test appointments throughout the seven-week period were included in the analysis. This means that 41 out of the 50 recruited patients were included in our analysis, 13 were in the 3D group, 10 were in the IMRT group, and 18 in the TOMO group. 

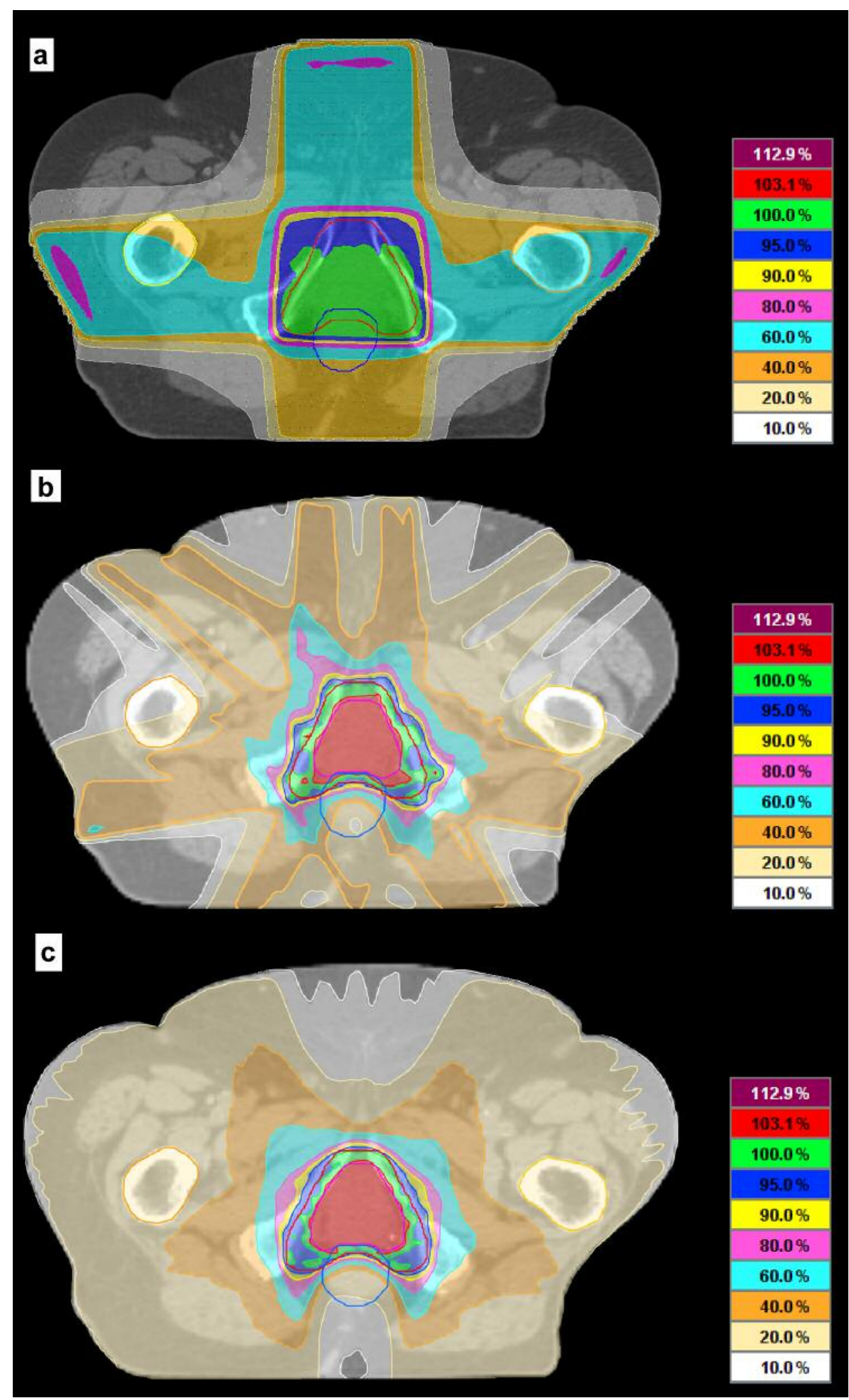

Figure 1. Typical transversal CT scan of the prostate region with transversal dose distribution of the applied radiotherapy depending on the used irradiation technique: (a) 3D, (b) IMRT, (c) TOMO. Planning target volume (PTV) is defined by the red line. Isodoses relative to the described cumulative dose (100\%) were shown; see also Table I. 
A

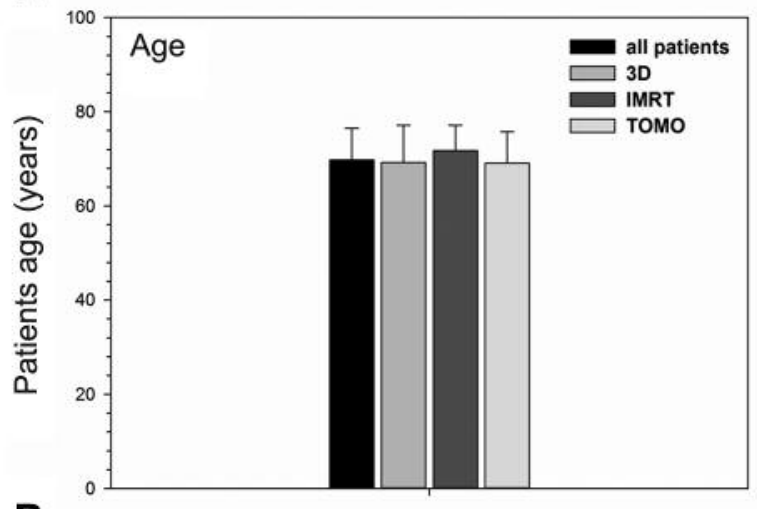

B

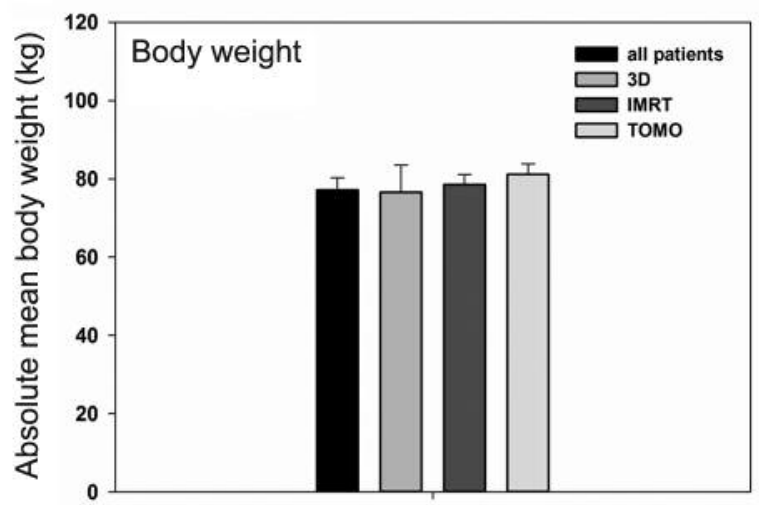

Figure 2. Patient age (a) and body weight (b).

Blood sample analyses were carried out using standardised methods in the University hospital to minimise variability and ensure comparability among samples. All samples were coded to prevent bias during manual scoring. The following data were determined: absolute numbers of leukocytes and thrombocytes per $\mathrm{ml}$ blood, and the concentration of haemoglobin in the blood $(\mathrm{mg} / \mathrm{dl})$. Laboratory-specific norm values for haemoglobin concentration ranged from $13 \mathrm{mg} / \mathrm{dl}$ to $17 \mathrm{mg} / \mathrm{dl}$. For leukocyte counts per $\mathrm{ml}$, the range was $4 \times 10^{6}$ to $10 \times 10^{6}$. The thrombocyte count per $\mathrm{ml}$ ranged from $150 \times 10^{6}$ to $400 \times 10^{6}$.

Body weight. The body weight of all patients was determined on the day of first treatment before the onset of radiotherapy. To calculate the total body volume, the following formula was used as published for male individuals: body volume $(\mathrm{l})=$ body weight $(\mathrm{kg}) \times 1.075(1 / \mathrm{kg})(18)$.

Statistical analysis. The statistics were processed in Sigma Plot $10.0^{\circledR}$. Student's $t$-tests were used, and $p$-values $<0.05$ were defined as statistically significant.

\section{Results}

The different treatment groups had almost identical median ages: 70.0 years for 3D, 70.5 years for IMRT, and 69.5 years for TOMO. Measurements of the median total body weight
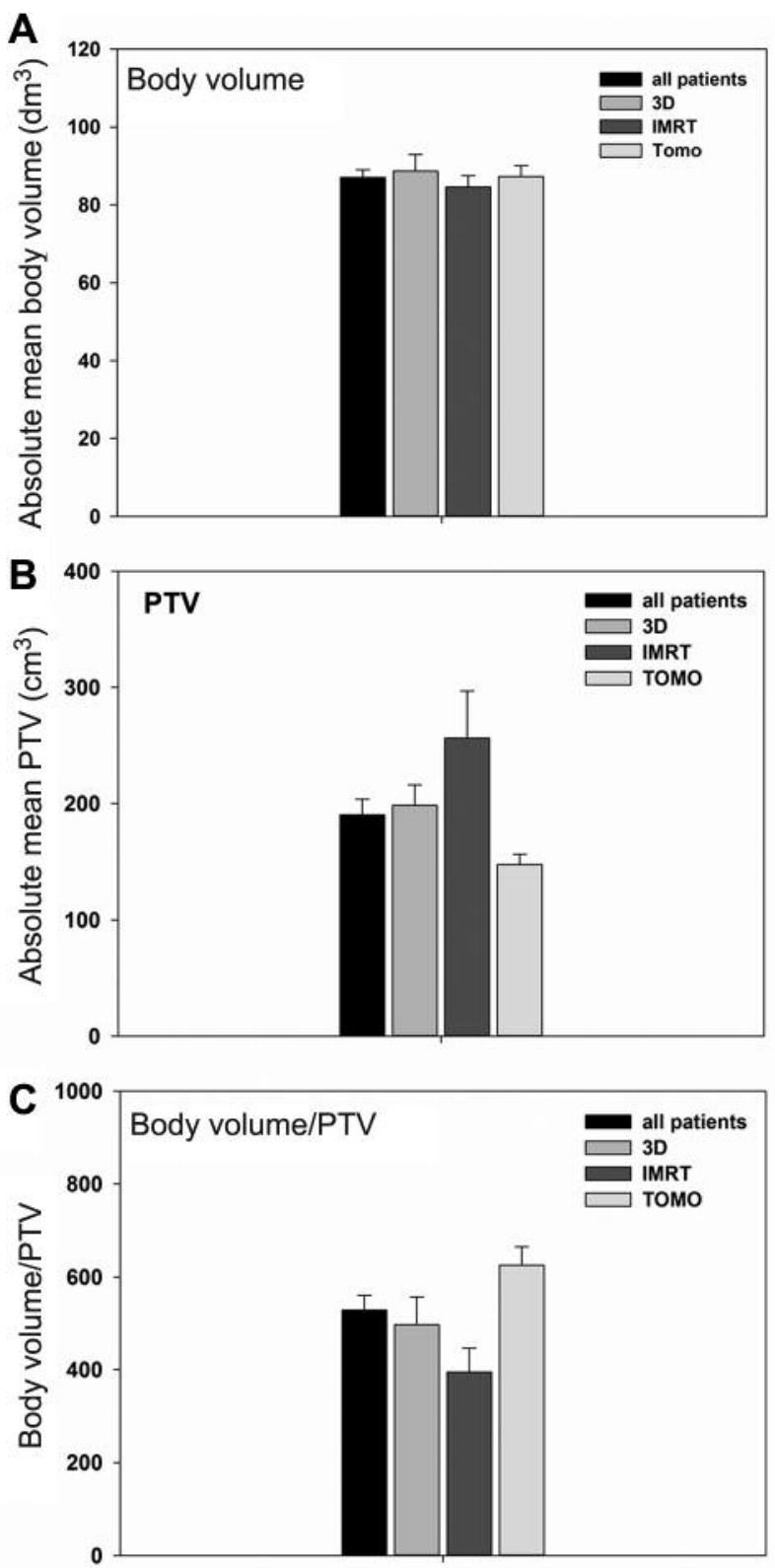

Figure 3. Patient body volume (a), planning target volume (PTV) (b)and body volume/PTV $(c)$.

also showed similar results for all three groups: $79.5 \mathrm{~kg}$ for $3 \mathrm{D}$, $79.0 \mathrm{~kg}$ for IMRT and $80.5 \mathrm{~kg}$ for TOMO. The median total body weight for all patients was $80.0 \mathrm{~kg}$ (see also Figure 2).

Correspondingly, there was no relevant difference in the median total body volume (TBV) between the groups: $3 \mathrm{D}$ at $86.00 \mathrm{dm}^{3}$, IMRT at $85.46 \mathrm{dm}^{3}$, and TOMO at $89.76 \mathrm{dm}^{3}$. The median TBV of all patients was $86.00 \mathrm{dm}^{3}$. Mean values are shown in Figure 3. The PTV depends on the individual's anatomy and prostate size. We calculated the following median values for the different groups: $185.50 \mathrm{~cm}^{3}$ for $3 \mathrm{D}$, 
A

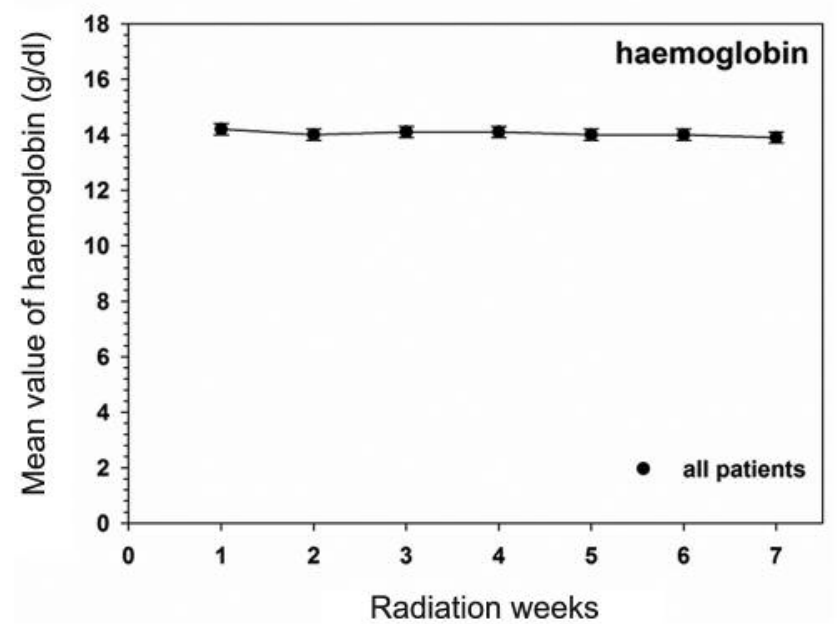

C

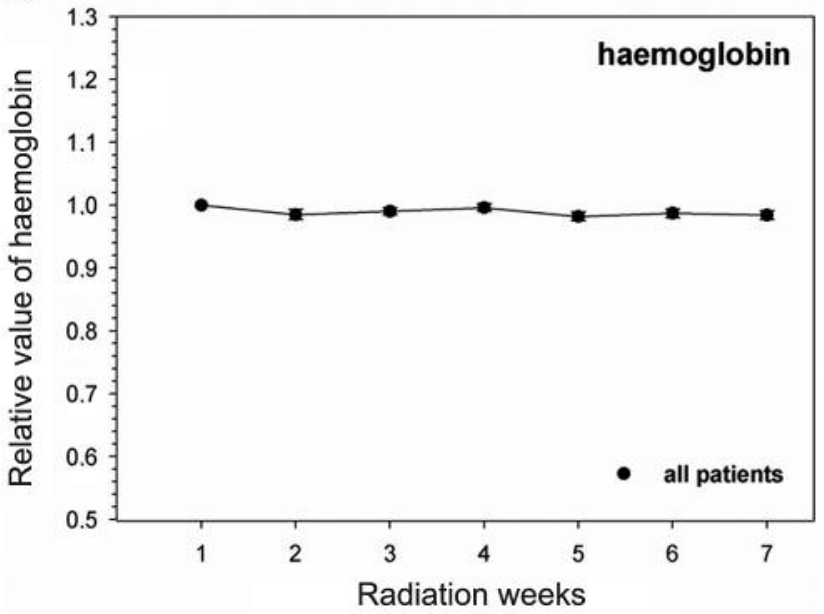

B

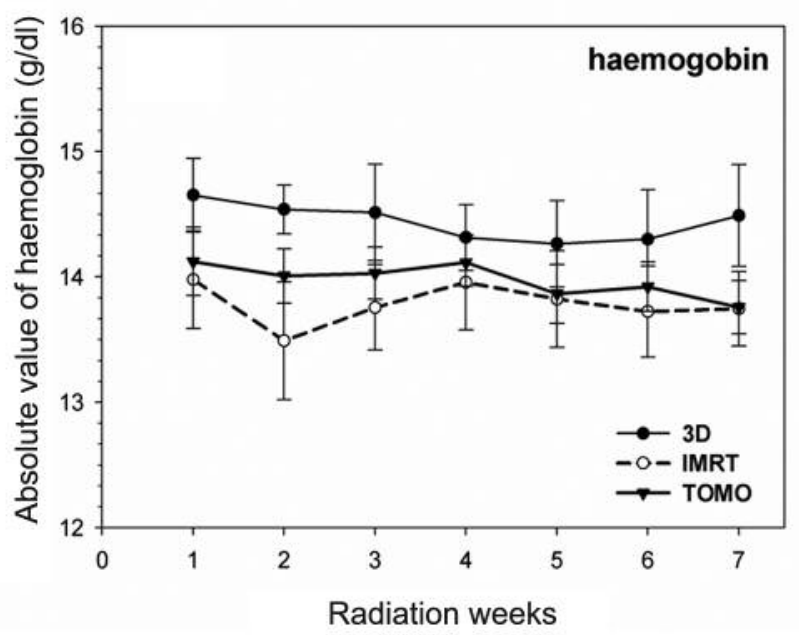

D

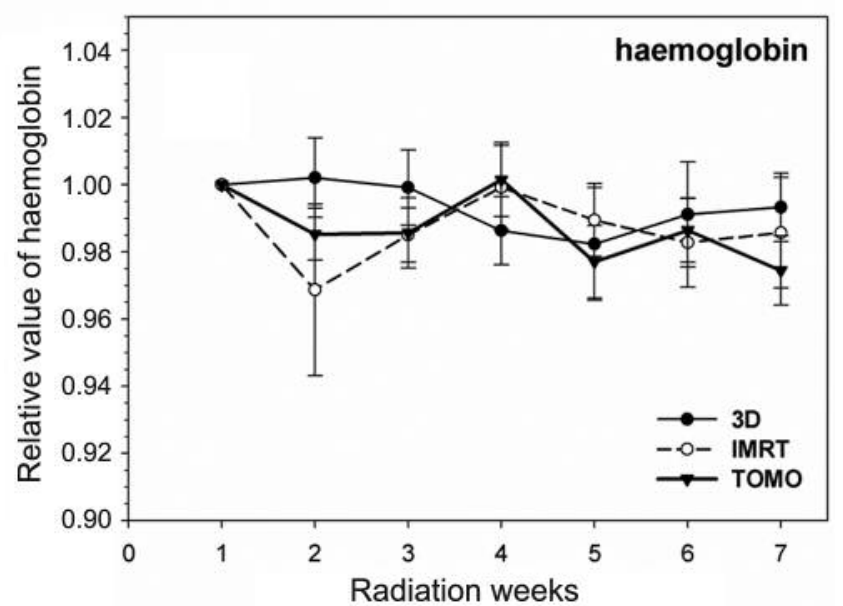

Figure 4. (a) Median absolute concentration of hemoglobin in the blood (g/dl) across all study patients, shown over the course of 7 weeks of conventional fractionated radiation therapy. (b) Absolute results split by delivery mode used for radiation. (c) Relative measurements averaged for all patients. (d) Relative measurements split by delivery mode used for radiation. Standard errors are shown.

$228.15 \mathrm{~cm}^{3}$ for IMRT, and $135.89 \mathrm{~cm}^{3}$ for TOMO. The median PTV calculated for all patients was $171.29 \mathrm{~cm}^{3}$. Mean values are shown in Figure 3. The value of TBV/PTV was calculated for each patient and led to a median quotient value of 522 for all patients. Accordingly, the three studied groups yielded median quotients of a) 407 (3D), b) 402 (IMRT), and c) 645 (TOMO). The mean values are shown in Figure 3.

Blood measurements. Over the 7 weeks of radiation therapy to the prostate/prostatic fossa, the haemoglobin concentration $(\mathrm{g} / \mathrm{dl})$ and leukocyte and thrombocyte counts per $\mathrm{ml}$ were analyzed.

The estimated median absolute blood concentration of haemoglobin for all patients did not change in a statistically significant manner over the course of radiotherapy, nor were any statistically significant changes observed in any of the three subgroups (3D, IMRT, and TOMO). The same is true for the relative changes to the mean haemoglobin concentration (Figure 4).

A small, but statistically significant, decrease was observed in absolute mean leukocyte counts per $\mathrm{ml}$ blood when all the patients were considered after the first week of radiation: $7.5 \times 10^{6}$ (standard error $\left.+/-0.2 \times 10^{6}\right) v s .6 .6 \times 10^{6}\left(+/-0.2 \times 10^{6}\right)$, $p=0.05$. This difference was maintained constant from the onset and during the rest of the treatment period. In the $7^{\text {th }}$ week of treatment, the mean count was $6.3 \times 10^{6}(+/-$ $0.2 \times 10^{6}$ ). Regarding the three subgroups, it is evident that this effect was mainly caused by the two IMRT groups (IMRT and TOMO). In the TOMO group leukocytes decreased from $7.03 \times 10^{6}\left(+/-0.3 \times 10^{6}\right)$ in the $1^{\text {st }}$ week to 
A

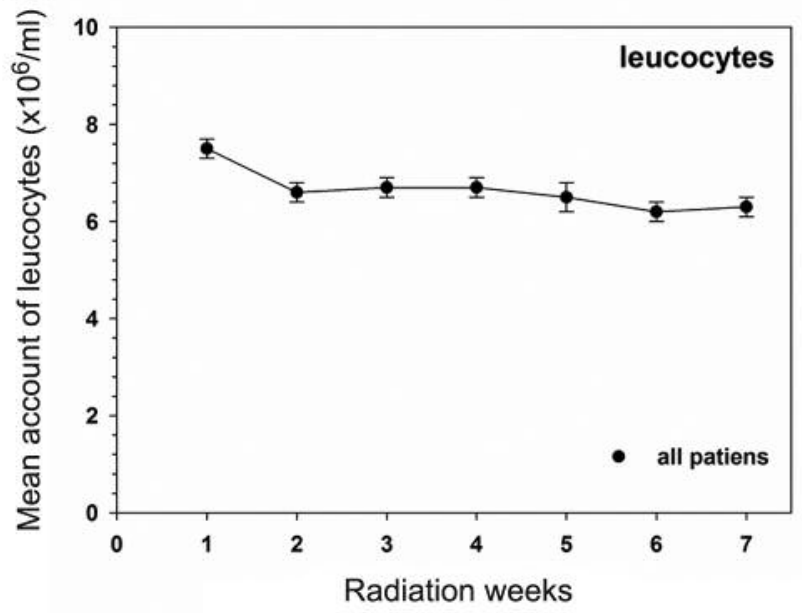

C

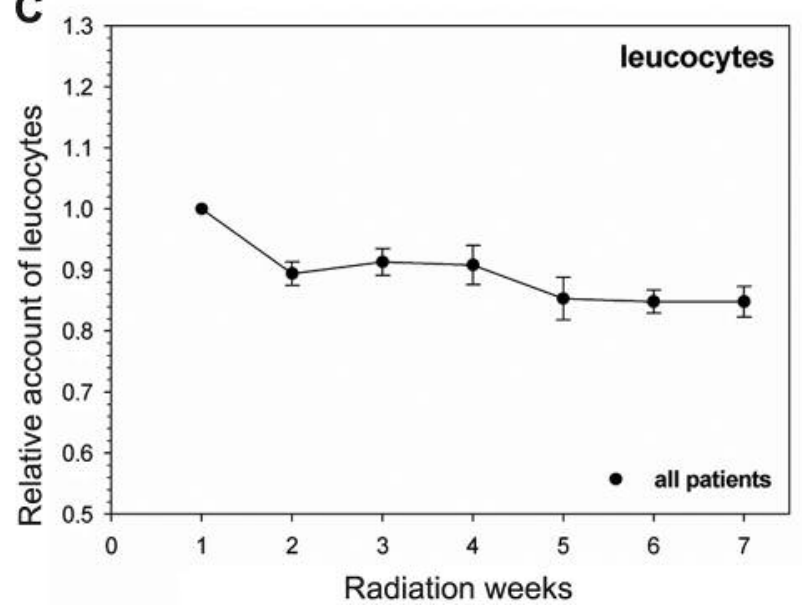

B
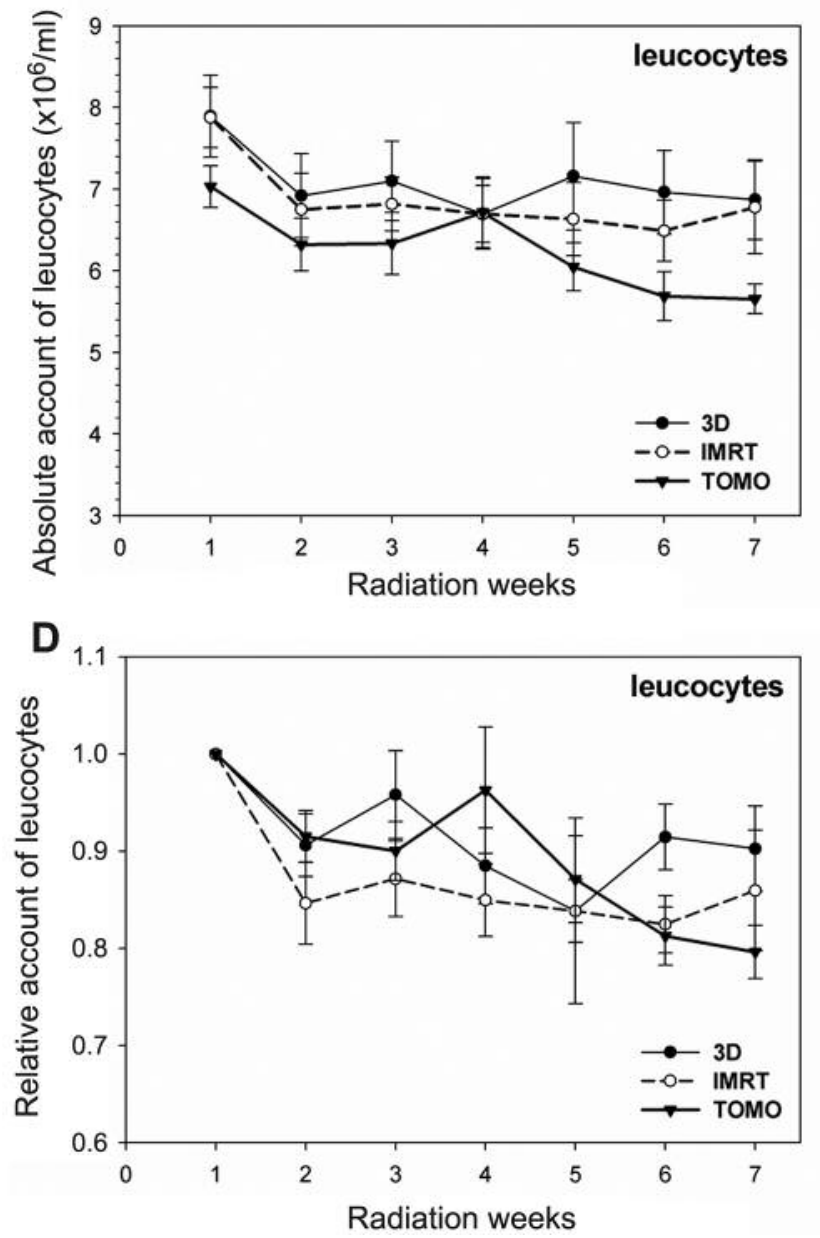

Figure 5. (a) Median absolute leukocyte count per ml blood across all study patients, shown over the course of 7 weeks of conventional fractionated radiation therapy. (b) Absolute results split by delivery mode used for radiation. (c) Relative measurements averaged for all patients. (d) Relative measurements split by delivery mode used for radiation. Standard errors are shown.

$5.65 \times 10^{6}\left(+/-0.2 \times 10^{6}\right)$ in the $7^{\text {th }}$ week of radiation, $(p=0.05)$. Furthermore, in the $7^{\text {th }}$ week of radiation, the TOMO group displayed a statistically significant lower mean leukocyte count compared to the 3D group $(p=0.05)$.

This slight decrease never led to leukopenia, (i.e. in counts $<4 \times 10^{6} / \mathrm{ml}$ ) (Figure 4). A similar pattern was observed in the case of the mean relative leukocyte count (Figure 5).

Neither the absolute nor the relative mean thrombocyte counts were statistically significant over the course of radiation therapy in the case of the group as a whole or the subgroups (Figure 6).

\section{Discussion}

The ages of the subgroups of participants (3D, IMRT, TOMO) were almost identical and the total body volumes were comparable. The TBV/PTV values were larger in the TOMO group than in the 3D or IMRT groups (645 vs. 407 and 402). The trend in blood counts across the entire patient group yielded no statistically significant drops in the haemoglobin concentration or thrombocyte count over the course of radiation therapy to the prostate region. There were also no statistically significant changes within the three subgroups. A small, but statistically significant, decrease in leukocyte count was observed for the entire group. This was particularly noticeable in the TOMO group although the median PTV was somewhat smaller for this group. This meant that the TBV/PTV value was larger than for the other groups. At no point during the course of radiation, however, was this decrease clinically relevant, nor did it result in any cases of leukocytopenia (grades 1-4). This prospective study, therefore, showed that (primary or additive) conventional 
A
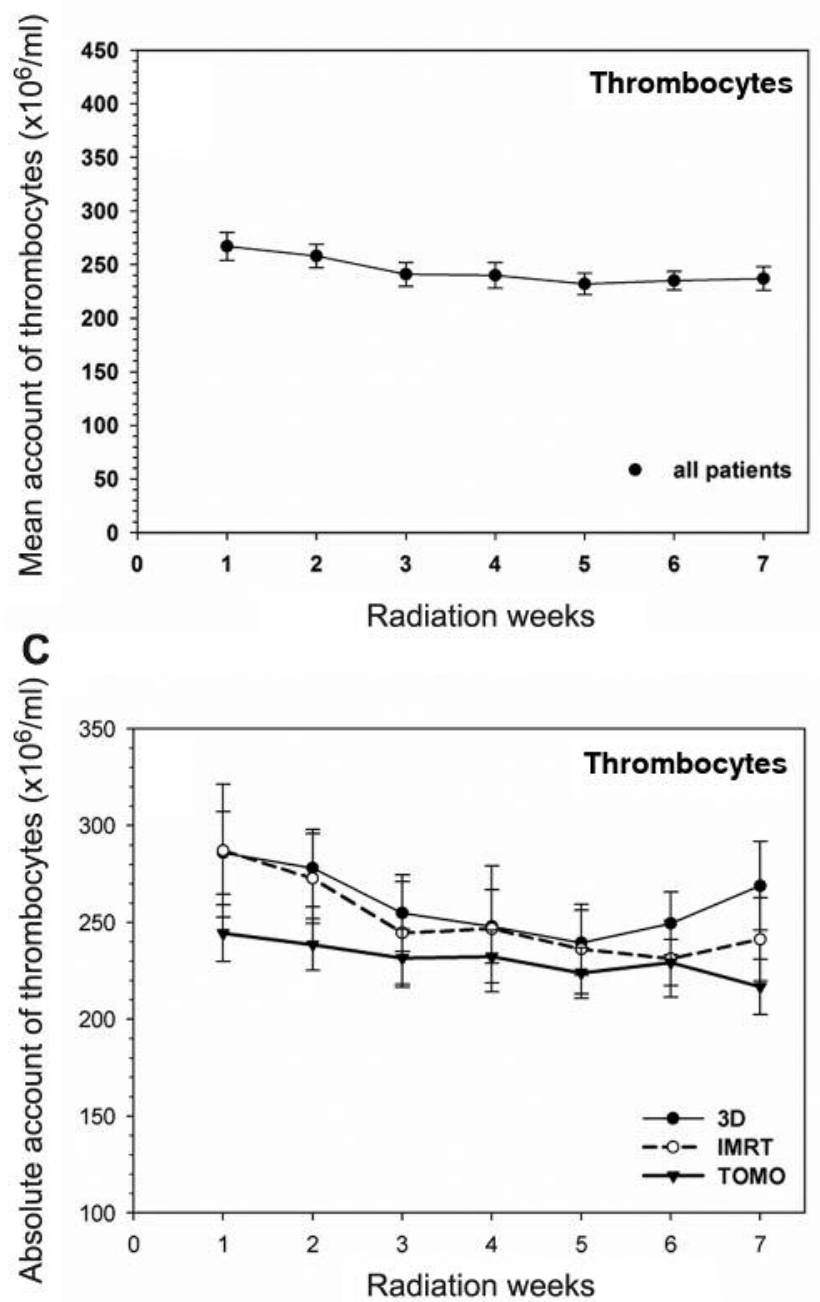

B

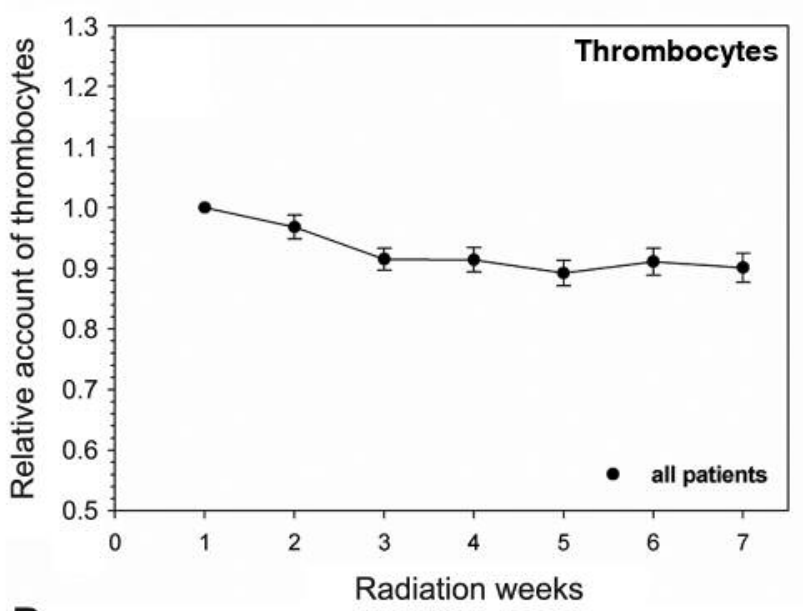

D

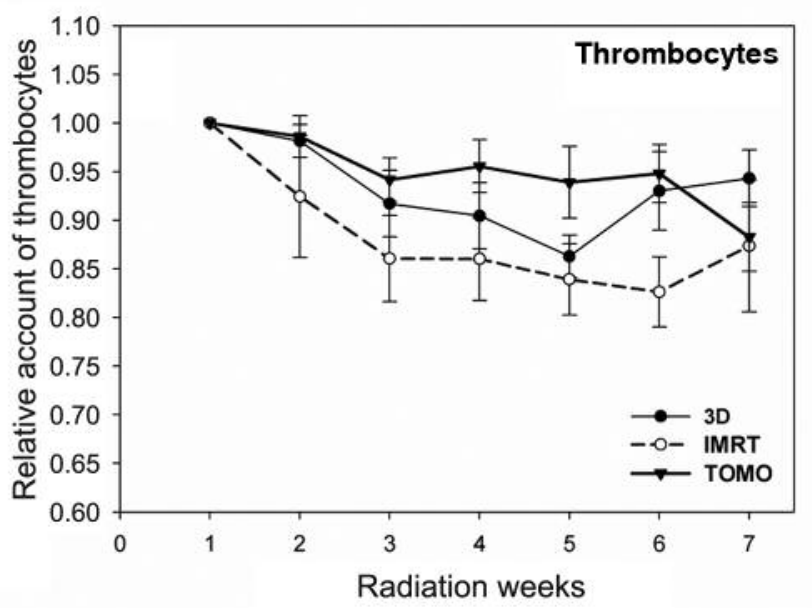

Figure 6. (a) Median absolute thrombocyte count per ml blood across all study patients, shown over the course of 7 weeks of conventional fractionated radiation therapy. (b) Relative measurements averaged for all patients. (c) Absolute results split by delivery mode used for radiation. (d) Relative measurements split by delivery mode used for radiation. Standard errors are shown.

fractionated radiotherapy for localised prostate cancer delivered by $3 \mathrm{D}$, or by one of two different IMRT techniques (IMRT or TOMO), did not lead to any clinically relevant changes to the partial blood count over the course of treatments. This finding is important because IMRT is associated with a higher transversal integral dose in the normal tissue compared to $3 \mathrm{D}$ conformal $\mathrm{RT}$ in the irradiation of the pelvis (19). Tomotherapy causes increased low-dose exposure and a relatively moderate medium-dose exposure of the surrounding normal tissue (12).

A study by Brixey et al. in 2002 examined the impacts of radiation therapy and radiochemotherapy for gynaecological tumours on blood counts. The study used IMRT and 3D conformal radiation therapy on the entire pelvic lymphatic drainage system. It was shown that $10 \%$ of patients who received only radiation therapy displayed grade 3 leukopenia (or a higher grade). No statistically significant difference was observed between the two delivery modes. As expected, parallel chemotherapy led to a statistically significant increase in the number of cases of leukopenia (13).

It has also been demonstrated that in post-surgical radiation therapy for cervix carcinoma, the reduction of size of the irradiated area from $16 \times 18 \mathrm{~cm}$ (whole pelvis) to $14 \times 10 \mathrm{~cm}$ (pelvis minor) led to statistically significant decreases in the rate of leukocytopenia (grades 1-3, from $80.5 \%$ to $52.2 \%)(20)$.

In 2016, Sini et al. published a study that showed that the IMRT of the prostate, including the pelvic lymphatic drainage 
system, was commonly associated with a statistically significant reduction in the number of white blood cells of the order of $\sim 30 \%$. A retrospective article by Pinkawa et al. (15) compared prostate cancer treatments using radiation therapy to the pelvis $v s$. the prostatic fossa. The delivery mode used was conventional fractionated five-field IMRT. Irradiation of the pelvis led to a significantly higher rate of grade 2 leukopenia $(15 \%$ vs. $2 \%)$. In our prospective study of conventional fractionated radiation therapy of the prostate region delivered by any of the three studied methods (3D, IMRT, or TOMO), indicated that treatments did not lead to any cases of clinically relevant leukocytopenia (grades 1-4), thrombocytopenia, or anaemia.

In the case at which only the prostate/prostate-region was treated, the study by Sanguineti et al. (16) compared leukocyte counts by following hypofractionated versus conventional fractionated radiation treatments for localised prostate cancer. Treatment was delivered using the 3D conformal mode. The conventional-fractionated group displayed a statistically significant drop in their leukocyte count $(20 \%$ vs. $15 \%)$. The prospective study presented herein led to a maximum drop of $15 \%$ in week 5 of radiation therapy in the 3D group. In our study the maximum overall drop in leukocyte count was observed in the TOMO group in week 7 of the treatment. Absolute counts remained at approximately $4 \times 10^{6} / \mathrm{ml}$ at all times. These values did not qualify as true leukocytopenia cases.

Based on the results of this study, we may infer that regular blood counts are not necessary during courses of radiation therapy of only the prostate/prostatic fossa when the PTV was $<230 \mathrm{~cm}^{3}$, regardless of which delivery mode was used (3D, IMRT, or TOMO). Regular blood counts should continue to be carried out when risk factors are present, such as immunodeficiency or clinical symptoms.

Discussion may also be warranted as to whether regular blood counts are required for target volumes $<230 \mathrm{~cm}^{3}$ localised in any part of the patients' body in case of absent risk factors. The area for irradiation in this study is located within the pelvic area, i.e. the most significant area in the body for haematopoiesis. Therefore, the risk of blood count changes should not be greater in other regions when the tumour volume is the same. For example, this may be the case when singular bone metastases are irradiated. As well relevant changes to the blood count is not supposable when hypo-fractionated radiation regimes are used for radiotherapy of the prostate gland with daily single dose upper than $2.5 \mathrm{~Gy}$, because treatment timeperiod is shortened. However, additional clinical research is required to confirm or disapprove these assertions.

For many patients, venipuncture is an unpleasant and sometimes painful procedure. Possible side-effects include bruising, infections (abscess, phlebitis, erysipelas), and damages to cutaneous nerves. Therefore, venipuncture should only be carried out when is medically necessary, i.e. when it provides information that reliably leads to a medical or therapeutic consequence. Based on the results of this study, blood tests are unnecessary in this case.

From a financial perspective, the health service costs can be reduced if weekly blood withdrawals and lab analyses are stopped in the cases of localised prostate cancer patients $\left(\mathrm{PTV}<230 \mathrm{~cm}^{3}\right)$ who are undergoing radiation therapy and who do not display any risk factors. To calculate the potential savings, we must consider the cost of materials (needles, blood collection tubes, swabs and plasters), lab analyses, and the man hours for the medical staff drawing the blood. On this basis, the cost of an individual blood test is approximately $15 \mathrm{EUR}$, i.e. $100 \mathrm{EUR}$ per patient for the total course of seven tests.

Each year, around 60,000 new cases of prostate cancer are diagnosed in Germany (21). According to data recorded by the German Cancer Society (DKG), approximately $20 \%$ of these patients undergo radiation therapy of the prostate region at some point in their cancer history (22). This results in approximately 10,000 courses of treatments per year in Germany. Weekly blood counts over 7 weeks, each incurring costs of 15 EUR, adds annual costs to the German health system of up to $\sim 1,000,000$ EUR annually.

Based on these figures, one may extrapolate an approximate cost for the 1.1 million new diagnoses made annually around the world (23). An additional option for consideration would be to carry out just one blood count at the onset of the conventional fractionated radiation therapy of the prostate region and continue with additional blood counts during the course of radiation treatment only if a) the initial test yield abnormal results, or b) the patient presents risk factors, or c) the patient shows clinical infection symptoms.

Irradiation of the prostate region for localised prostate cancer is very commonly indicated in Germany, Europe, and around the world. This prospective study may thus contribute to the optimisation of treatment delivery.

\section{Conclusion}

Regardless of the delivery mode used, localised fractionated irradiation of the prostate region did not cause any clinically relevant changes to the blood count in this study. Accordingly, these findings question the necessity of regular blood counts during courses of radiation therapy of the prostate region for patients who do not display any risk factors.

\section{Conflicts of Interest}

The Authors declare that there are no conflicts of interest.

\section{Authors' Contributions}

F.Z., K.H. and H.H. initiated and supervised the project. B.S. and F.Z. collected data and carried out the data analysis. F.Z., B.S., 
H.H., P.H., and J.D. interpreted the clinical data and were responsible for creating figures. F.Z., H.H. and P.H. wrote the manuscript with input from all authors. All Authors were involved in manuscript revisions.

\section{References}

1 Hama Y and Kaji T: Long-term follow-up results of ct-guided daily adaptive radiation therapy for localized prostate cancer Anticancer Res 38: 5959-5962, 2018. PMID: 30275225. DOI: 10.21873/anticanres.12942

2 Ahmad P, Sana J, Slavik M, Gurin D, Radova L, Gablo NA, Kazda T, Smilek P, Horakova Z, Gal B, Hermanova M, Slampa $\mathrm{P}$ and Slaby O: MicroRNA-15b-5p predicts locoregional relapse in head and neck carcinoma patients treated with intensitymodulated radiotherapy. Cancer Genomics Proteomics 16: 139146, 2019. PMID: 30850365. DOI: 10.21873/cgp.20119

3 Makino T, Nakashima K, Iijima M, Kawaguchi S, Nohara T, Shigehara K, Izumi K, Kadono Y, Kumano T and Mizokami A: Health-related quality of life and toxicity after single-fraction highdose-rate brachytherapy with external beam radiotherapy for localized and locally advanced prostate cancer. Anticancer Res 39: 477-486, 2019. PMID: 30591498. DOI: 10.21873/anticanres.13137

4 Wiezorek T, Schwahofer A and Schubert K: The influence of different IMRT techniques on the peripheral dose: a comparison between sMLM-IMRT and helical tomotherapy. Strahlenther Onkol 185: 696-702, 2009. PMID: 19806336. DOI: 10.1007/ s00066-009-2005-9

5 Wiezorek T, Voigt A, Metzger N, Georg D, Schwedas M, Salz $\mathrm{H}$ and Wendt TG: Experimental determination of peripheral doses for different IMRT techniques delivered by a Siemens linac accelerator. Strahlenther Onkol 184: 73-79, 2008. PMID: 18259698. DOI: $10.1007 / \mathrm{s} 00066-008-1743-4$

6 Wiezorek T, Georg D, Schwedas M, Salz H and Wendt TG: Experimental determination of peripheral photon dose components for different IMRT techniques and linear accelerators. Z Med Phys 19: 120-128, 2009. PMID: 19678527. DOI: 10.1016/j.zemedi.2009.01.008

7 Chofor N, Harder D, Willborn KC and Poppe B: Internal scatter, the unavoidable major component of the peripheral dose in photon-beam radiotherapy. Phys Med Biol 57: 1733-1743, 2012. PMID: 22398213. DOI: 10.1088/0031-9155/57/6/1733

8 Vanasek J, Odrazka K, Dolezel M, Kolarova I, Jarkovsky J, Pavlik T, Hlavka A and Dusek L: Statistical analysis of dosevolume profiles and its implication for radiation therapy planning in prostate carcinoma. Int J Radiat Oncol Biol Phys 86: 769-776, 2013. PMID: 23628133. DOI: 10.1016/j.jirobp.2013.03.018

9 Onal C, Efe E, Guler OC and Yildirim BA: Dosimetric comparison of sequential versus simultaneous-integrated boost in early-stage breast cancer patients treated with breastconserving surgery. In Vivo 33: 2181-2189, 2019. PMID: 31662554. DOI: 10.21873 /invivo. 11720

10 Wright JD, St Clair CM, Deutsch I, Burke WM, Gorrochurn P, Sun $\mathrm{X}$ and Herzog TJ: Pelvic radiotherapy and the risk of secondary leukemia and multiple myeloma. Cancer 116: 24862492, 2010. PMID: 20209618. DOI: $10.1002 /$ cncr. 25067

11 Zwicker F, Kirchner C, Huber PE, Debus J, Zwicker H and Klepper R: Breast cancer occurrence after low dose radiotherapy of non-malignant disorders of the shoulder. Sci Rep 9: 5301, 2019. PMID: 30923327. DOI: 10.1038/s41598-019-41725-w
12 Sini C, Fiorino C, Perna L, Noris Chiorda B, Deantoni CL, Bianchi M, Sacco V, Briganti A, Montorsi F, Calandrino R, Di Muzio N and Cozzarini C: Dose-volume effects for pelvic bone marrow in predicting hematological toxicity in prostate cancer radiotherapy with pelvic node irradiation. Radiother Oncol 118: 79-84, 2016. PMID: 26702990. DOI: 10.1016/j.radonc.2015.11.020

13 Brixey CJ, Roeske JC, Lujan AE, Yamada SD, Rotmensch J and Mundt AJ: Impact of intensity-modulated radiotherapy on acute hematologic toxicity in women with gynecologic malignancies. Int J Radiat Oncol Biol Phys 54: 1388-1396, 2002. PMID: 12459361. DOI: 10.1016/s0360-3016(02)03801-4

14 Cozzarini C, Noris Chiorda B, Sini C, Fiorino C, Briganti A, Montorsi $\mathrm{F}$ and Di Muzio N: Hematologic toxicity in patients treated with postprostatectomy whole-pelvis irradiation with different intensity modulated radiation therapy techniques is not negligible and is prolonged: Preliminary results of a longitudinal, observational study. Int J Radiat Oncol Biol Phys 95: 690-695, 2016. PMID: 27131081. DOI: 10.1016/j.ijrobp.2016.01.022

15 Pinkawa M, Djukic V, Klotz J, Petz D, Piroth MD, Holy R and Eble MJ: Hematologic changes during prostate cancer radiation therapy are dependent on the treatment volume. Future Oncol 10: 835-843, 2014. PMID: 24799064. DOI: 10.2217/fon.13.237

16 Sanguineti G, Giannarelli D, Petrongari MG, Arcangeli S, Sangiovanni A, Saracino B, Farneti A, Faiella A, Conte M and Arcangeli G: Leukotoxicity after moderately Hypofractionated radiotherapy versus conventionally fractionated dose escalated radiotherapy for localized prostate Cancer: a secondary analysis from a randomized study. Radiat Oncol 14: 23, 2019. PMID: 30700317. DOI: 10.1186/s13014-019-1223-2

17 Schlegel W and Kneschaurek P: Inverse radiotherapy planning. Strahlenther Onkol 175: 197-207, 1999. PMID: 10356608. DOI: $10.1007 / \mathrm{bf02742396}$

18 Vickery SR, Cureton KJ and Collins MA: Prediction of body density from skinfolds in black and white young men. Hum Biol 60: 135-149, 1988. PMID: 3371953.

19 Salz H, Eichner R and Wiezorek T: Does IMRT increase the peripheral radiation dose? A comparison of treatment plans 2000 and 2010. Z Med Phys 22: 6-12, 2012. PMID: 21530197. DOI: 10.1016/j.zemedi.2011.03.004

20 Ohara K, Tsunoda H, Satoh T, Oki A, Sugahara S and Yoshikawa $\mathrm{H}$ : Use of the small pelvic field instead of the classic whole pelvic field in postoperative radiotherapy for cervical cancer: reduction of adverse events. Int J Radiat Oncol Biol Phys 60: 258-264, 2004. PMID: 15337564. DOI: 10.1016/j.jirobp.2004.02.023

21 Interdisziplinäre Leitlinie der Qualität S3 zur Früherkennung, Diagnose und Therapie der verschiedenen Stadien des Prostatakarzinoms, Langfassung. Available at: https://www. leitlinienprogramm-onkologie.de/fileadmin/user_upload/ Downloads/Leitlinien/Prostata_5_0/LL_Prostatakarzinom_Langv ersion_5.1.pdf

22 Kennzahlenauswertung 2018 - Deutsche Krebsgesellschaft (German Cancer Association). Available at: https://www. krebsgesellschaft.de/jahresberichte

23 Prostate cancer guidelines of the der European Association of Urology (2014). Available at: http://uroweb.org/guideline/ prostate-cancer/

Received December 19, 2019

Revised January 30, 2020

Accepted February 3, 2020 